\title{
DEVELOPMENT OF A COMPUTER MODEL FOR PREDICTION OF COLLISION RESPONSE OF A RAILROAD PASSENGER CAR
}

\author{
Steven W. Kirkpatrick and Robert A. MacNeill \\ Applied Research Associates, Inc. \\ 2672 Bayshore Parkway, Suite 1035 \\ Mountain View, CA 94043
}

\begin{abstract}
The paper describes the development of a detailed finite element model that is capable of predicting the response of a rail passenger car to collision conditions. This model was developed to predict the car crush, the three-dimensional gross motions of the car, and the vertical, lateral, and longitudinal accelerations experienced by the car during collisions.

The finite element model developed was for a Pioneer passenger coach car. This vehicle was used in a single car impact test. The model was then used to simulate the test and the results are compared to the test data.
\end{abstract}

\section{INTRODUCTION}

There is an ongoing research program in the United States to investigate and improve rail equipment crashworthiness. As part of this effort, computer models have been developed and applied to determine the crash response of rail equipment. Comparison of the model predictions with full-scale test results is required to validate the models. A corresponding series of full-scale rail vehicle crash tests are being performed as part of the rail crashworthiness research effort. The first full-scale test, an impact of a single passenger coach car into a fixed wall, was conducted at the Transportation Technology Center in Pueblo, Colorado on November 16, 1999. The test consisted of a single Pioneer car traveling at $35.1 \mathrm{mph}$ when it impacted the wall [14].

The objectives of this test were to determine the failure modes of the major structural components, to measure the gross motions of the car, to measure the force/crush characteristic, and to evaluate selected occupant protection strategies. The condition of the test vehicle both before and after the collision is shown in Figure 1.

The objective of this effort was to develop a detailed finite element model that is capable of predicting the rail passenger car response to collision conditions. The crash responses to be modeled include the car crush history, the structural deformation modes, and the gross motions of the car in the vertical, lateral, and longitudinal directions. The final objective was to use the model to simulate the collision test and compare the results to the test data.
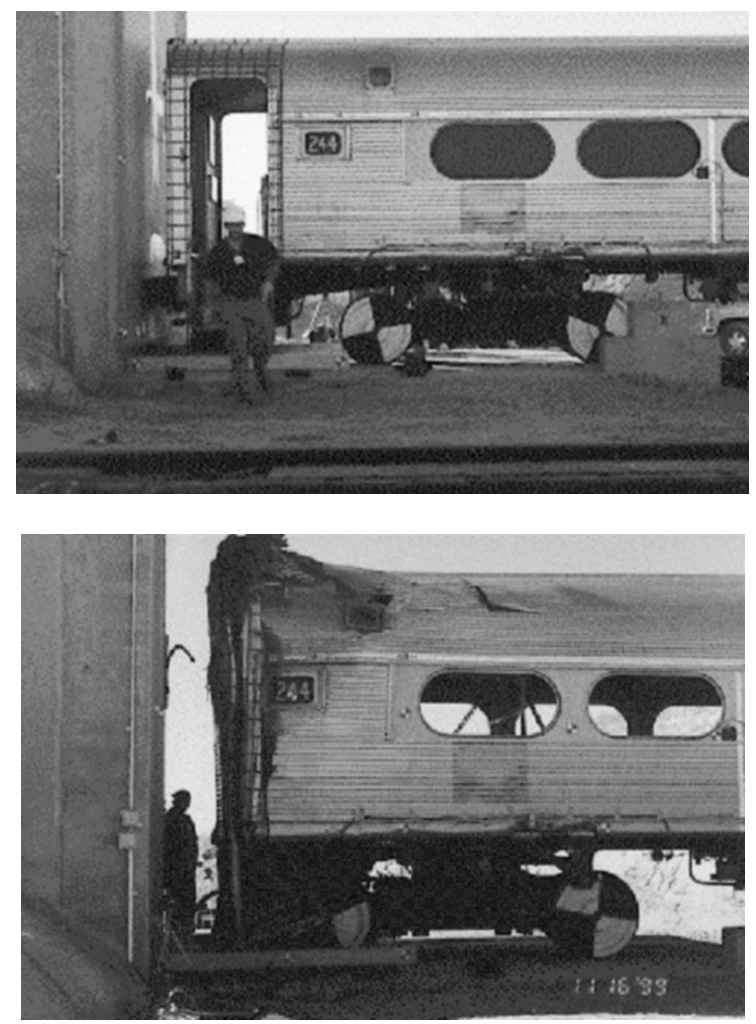

Figure 1. Photographs of the Single Coach Car Crash Test.

As part of this effort, the test data was analyzed and used to determine which features of the rail vehicle that needed to be included in the model to accurately simulate the collision response. The crash response to the 35-mph impact produced 
an average longitudinal crash deceleration of approximately $7 \mathrm{~g}$ and a peak crush distance on the front end of the vehicle of approximately 66 inches. The vehicle rebounded from the wall at a velocity of approximately 3-mph. The duration of the longitudinal crash pulse was approximately 0.25 second. Additional details from the data analysis and vehicle postmortem are given in References 5 and 6.

The vertical and lateral motions of the vehicle were small compared to the longitudinal response. The vertical motion of the car body consisted of an upward lifting of the forward car body with a maximum displacement of approximately 5.5 inches. The vertical car body motion consists of both an extension of the secondary suspension to its limit of approximately 2.5 inches and a subsequent lift of the front truck off the rails of approximately 3 inches. The analysis of the lateral accelerometer measurements indicated a rotation with the front end of the occupant compartment displaced approximately 9 inches to the left side and the rear of the car displaced approximately 2 inches to the right.

Inspection of the vehicle showed that the draft sill was the dominant structural component and was expected to dissipate a significant fraction of the collision energy.

\section{MODEL DEVELOPMENT}

The initial approach for the model development was to modify the existing model of an Amfleet car [7] to simulate a Pioneer car. There are many structural similarities in the design of the Amfleet and Pioneer cars. However, after consideration of the differences between the two vehicles, it was determined that the best approach would be to develop a new model rather than modify the existing model. This decision was based on the large number of changes required to produce a high fidelity model of the Pioneer car that would be capable of simulating both the car crush and gross vehicle motions.

A detailed LS-DYNA finite element model was developed for the Pioneer coach car [8]. The model was developed to include the appropriate geometric, material, and inertial properties to represent the Pioneer car used in the impact test performed at TTCI. The model has a higher fidelity description of the vehicle structures than previous models. Significant differences include detailed descriptions of the draft gear and vehicle suspension components. Modeling of the draft gear was important to obtain the correct collision load path into the draft sill, which is the largest structural member in the vehicle crush zone. Modeling of the secondary suspension was important to correctly model the coupling of the truck mass to the car body and for the modeling of the vehicle gross motions.

\section{CRUSH MODELING}

The approach in the model development was to try and maintain a relatively uniform mesh throughout the vehicle. An additional objective was to maintain a minimum element dimension of approximately 0.75 -inch. A few smaller elements were needed to create some components such as the traction rod connection bracket. However, mass scaling could be used at these locations to prevent them from adversely controlling the calculation time step.

The Pioneer coach car model developed is shown in Figure 2. The model has approximately 429,000 4-node shell elements and 87,000 8-node solid hexahedral (brick) elements. Several features of the model fidelity are apparent. First, the model is seen to have detailed descriptions of the draft gear and the suspension components. These were identified as important components that influence both the structural collapse mechanisms and the vehicle collision dynamics.

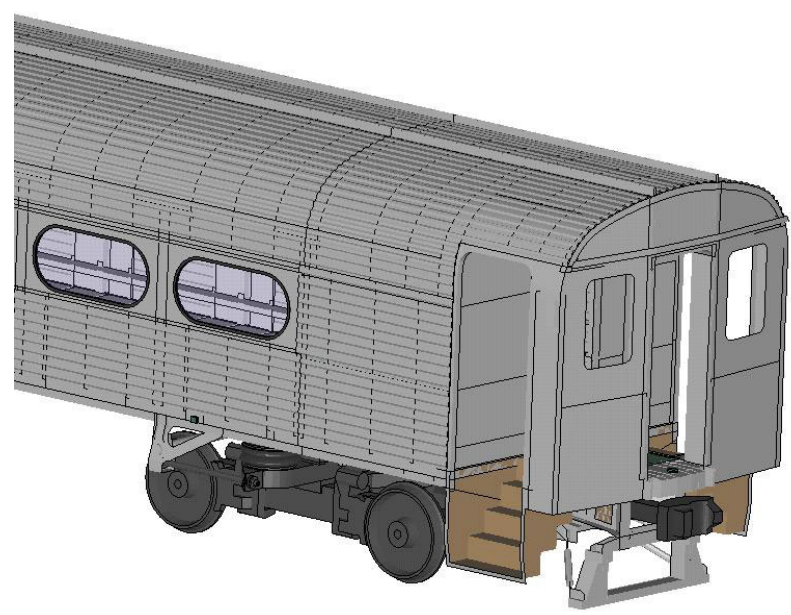

Figure 2. Pioneer Passenger Coach Car Model.

The superstructure components are also modeled with greater fidelity. The structural framework of the Pioneer car model with the outer sheathing removed is shown in Figure 3. The model was constructed to include the appropriate positions, spacing, and size of the structural stiffening.

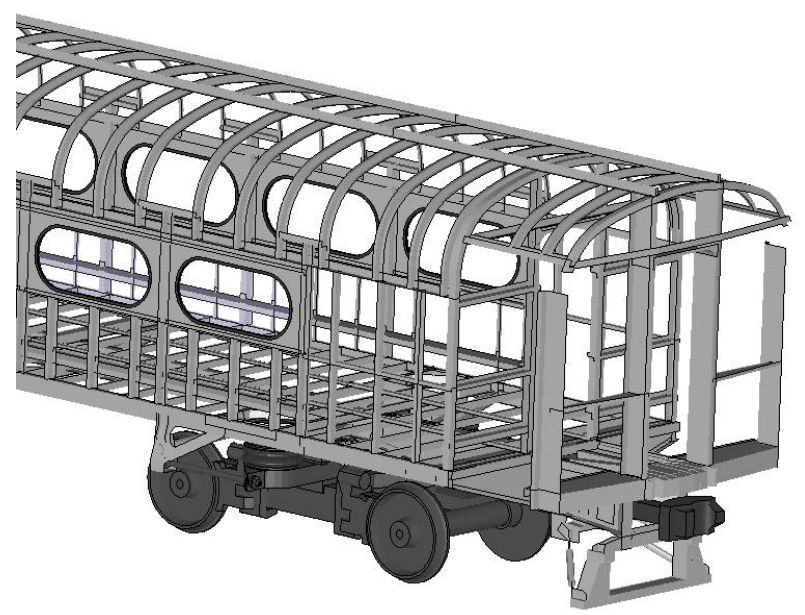

Figure 3. Model of Structural Framework for the Pioneer Passenger Coach Car.

An example of the improved fidelity in the structural modeling is the corrugated outer sheathing on the superstructure. In the previous model, a flat outer shell with a 
modified effective thickness was used to model the sheathing. The effective thickness was chosen to have the same longitudinal stiffness in the car body. However, in the crush zone, this approximation does not produce the same local bending stiffness and energy absorption. In the Pioneer coach car the corrugated sheathing was explicitly modeled. The only approximation was an increase in the corrugation wavelength to prevent the corrugation width from defining a minimum element dimension, which would control the time step size.

Many of the brick elements are contained within rigid bodies using the rigid material option in LS-DYNA. This significantly reduces the computational requirements for groups of elements that do not deform significantly during the collision. For example, the elements in each truck are grouped into a single rigid body. Similarly, the bolster beam is modeled as a rigid body. The connection between the bolster beam and truck is created using a rotational joint definition centered on the bolster beam center post.

Another feature included in the Pioneer car model is the ability for components and connections to fail. Three different modeling techniques were used in the development of the Pioneer model. First, the tied interface with failure algorithm was used to model structural connections in the crush zone. Second, a material constitutive model with failure was used for various components in the crush zone to allow for removal of elements that had exceeded the failure strain. Finally, a weld zone approximation with failure was added to the draft sill to allow for observed collapse mechanisms.

The tied with failure interface algorithms allowed for modeling connection failures of various components in the crush zone. The interface was primarily used to define the spot weld connection between the corrugated outer sheathing and the superstructure frame. The interface was also used to tie the superstructure to the under frame in the forward portion of the vehicle.

The interface algorithm prevents relative sliding or separation of adjacent tied element segments until a failure criterion is exceeded. After failure the interface acts like a traditional sliding-with-voids contact algorithm that prevents penetration between the two surfaces. The failure criterion used for the interface is a polynomial combination of the normal and shear force across an interface segment according to the relationship

$$
\left(\frac{F_{n}}{N}\right)^{2}+\left(\frac{F_{s}}{S}\right)^{2} \geq 1
$$

where $F_{n}$ and $F_{s}$ are the normal and shear forces across the interface segment and $N$ and $S$ are the normal and shear failure strengths.

The values of appropriate normal and shear interface strengths in the model will depend on a number factors including element size, interface penalties, spot weld spacing, and spot weld strengths. In the model, the values selected were estimated and then adjusted based on results of preliminary calculations.

The material constitutive algorithm with failure used in the train car model is material type 24 in LS-DYNA. The type 24 constitutive model specifies a piecewise linear isotropic elasticplastic material behavior. Most of the components in the crush zone are modeled with the type 24 material algorithm including the draft sill, collision post, corner posts, and the corrugated outer sheathing on the forward section of the car body.

Failure in the constitutive algorithm is controlled by a maximum effective plastic strain criterion. This is a simplified approach to modeling ductile failure of materials, but appropriate for the available information on the failure of the rail car materials. The parameters used for the plastic hardening modulus and failure strain were obtained by a series of material tests performed on specimens removed from various components on the test vehicle [5].

An additional modification was necessary to model the failure of the weldments in the draft sill. The weld failures in the draft sill were an important feature of the overall collapse mechanisms as shown in Figure 4. The preliminary modeling approach for the draft sill collapse was to use the constitutive model with failure. This approach did not include any special treatment of the weldments. However, with an element size on the order of an inch, the stress and strain concentrations at the weldment positions are not sufficiently captured in the draft sill to model the observed weldment failures.

The modified approach chosen for the final model was to define a row of elements along weldments in the draft sill with effective material properties. The weld zone elements are still approximately one inch wide. The yield stress in these weldment elements was reduced by $20 \%$ to account for the effects of the weld metal and stress concentrations around the weld. Additionally the failure strain of the weldment material was reduced by $40 \%$. The resulting approximations were found to allow the failure of the draft sill weldments in the model prior to bulk failure of the material in the draft sill structure, as observed in the test.

\section{COLLISION DYNAMICS MODELING}

An objective of the modeling in this program is to calculate both the vehicle crush behavior and the overall vehicle motions in the longitudinal, lateral, and vertical directions. This task is difficult because the mechanisms that result in vehicle vertical and lateral motions are not always fully understood. Potential mechanisms include the collapse of structures in the under frame in a mode that forms a ramp creating vertical lifting forces. An alternate mechanism is that the motions are produced when the center of the vehicle crush forces are not aligned with the CG of the vehicle. Thus, the collision forces produce a moment about the vehicle CG, resulting in vertical and lateral rotations of the car body during the crash. 


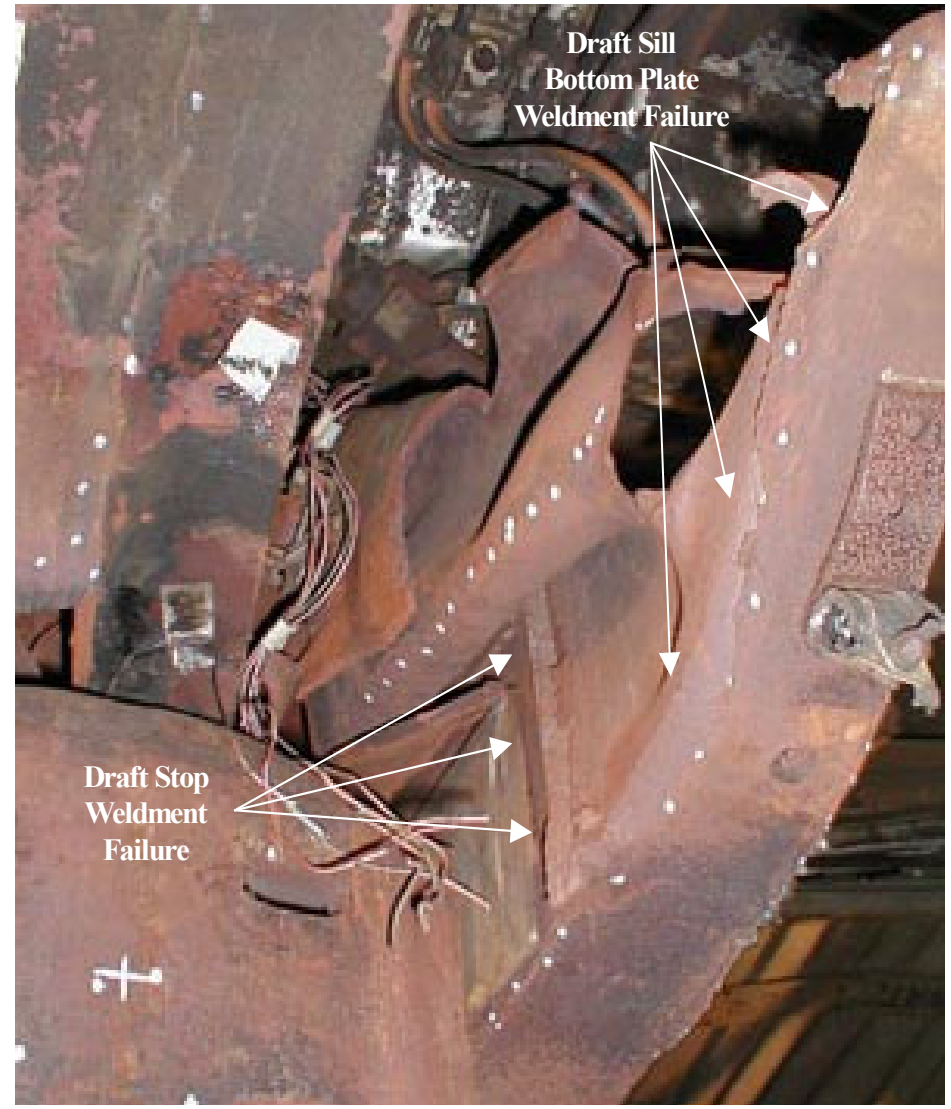

(a) observed draft sill weldment fractures

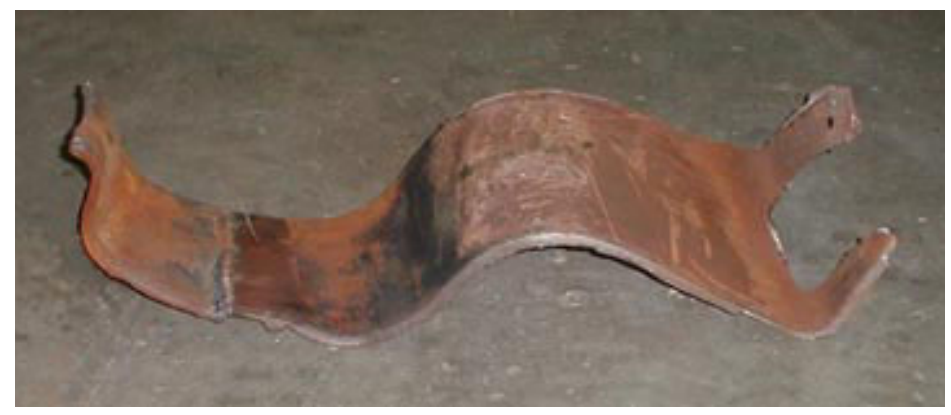

(b) draft sill bottom plate failure

Figure 4. Observed Weldment Fractures in the Collapse of the Draft Sill.

The approach used to model the collision dynamics was to first make sure that the crash behavior could be accurately modeled. These collision responses will produce the loads and deformations that act as the boundary conditions that control the resulting lateral and vertical vehicle motions. The second factor in the model development was to make sure that any of the features that could contribute to the collision dynamics were properly included in the model. These features included accurate reproduction of all inertial properties in the vehicle and modeling of the secondary suspension components.
The model of the truck and secondary suspension air springs is shown in Figure 5. The air spring consists of a cylindrical rubber baffle with an internal pressure of $48 \mathrm{psi}$ to suspend the weight of the car body. Another feature of the suspension that can be seen on the bolster beam in Figure 5 are the four hooks that catch on a set of retaining latch plates on the body bolster. These hooks prevent additional extension of the secondary suspension after the maximum travel of approximately 2.5 inches has been reached. This connection to the car body lifts the forward truck off of the rail during the collision.

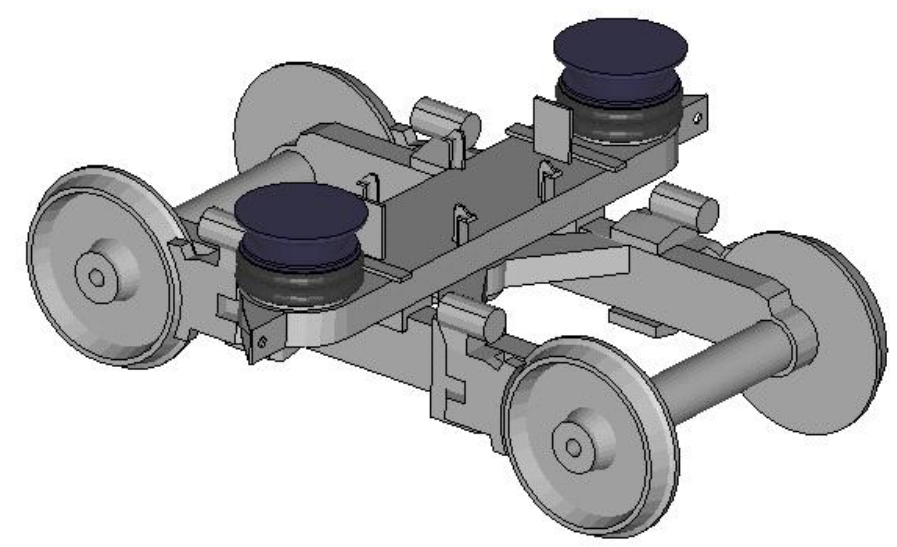

Figure 5. Model of the Truck and Secondary Suspension.

A difficulty in creating the suspension model was the determination of material properties for various components such as the air spring bellows and traction rod bushings. Initial estimates were made for these materials. The approach used to validate the suspension model was to simulate a bounce test on the suspension and compare to the measured vehicle secondary suspension bounce frequency of $1.19 \mathrm{~Hz}$. These bounce test simulations were used to investigate the influence of the various component material properties on the suspension behavior and to determine an appropriate set of material constants to accurately model the suspension stiffness. For computational efficiency the car body was replaced with a rigid mass of equivalent weight in the suspension bounce calculations. The model for the truck and suspension are identical to that used in the final Pioneer car model.

The results of a pair of bounce test simulations are shown in Figure 6. Initially, the estimates of properties for the suspension components produced a bounce frequency of 1.69 Hz. Initial adjustments to the air spring bellow stiffness lowered this frequency slightly. However, to match the bounce frequency, the properties of the traction rod bushings needed to be reduced. The final properties used in the suspension model resulted in a bounce frequency of $1.16 \mathrm{~Hz}$. This calculated bounce frequency for the suspension model is within $5 \%$ of the measured bounce frequency. 


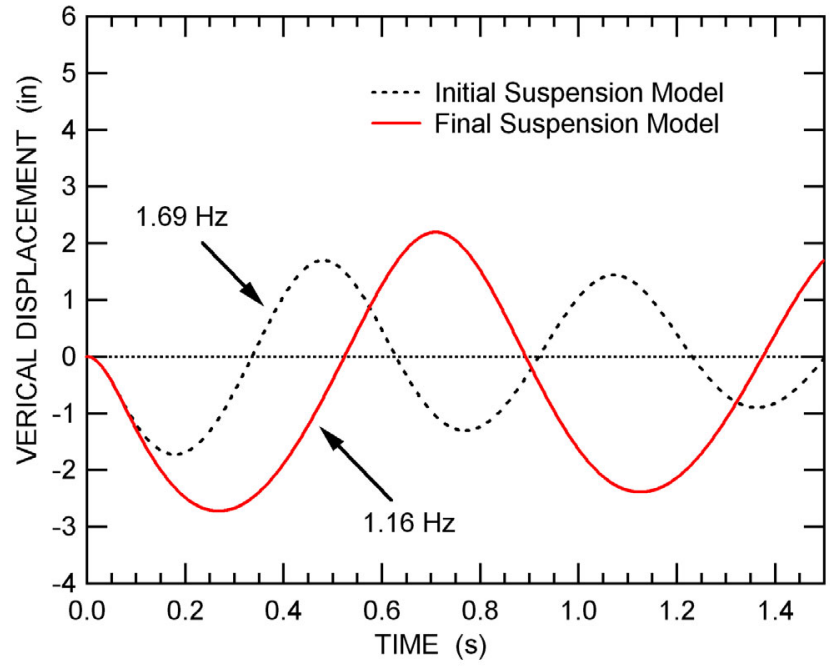

Figure 6. Analysis of the Secondary Suspension Bounce Frequency.

\section{SIMULATION OF THE CRASH TEST}

The Pioneer car model was used to simulate the crash response for the $35-\mathrm{mph}$ impact into a rigid wall. The measured and calculated longitudinal displacements are compared in Figure 7. The comparison shows good agreement between the measured and calculated car body crush. The calculated maximum crush distance was 65 inches, which agrees very closely with the measure crush.

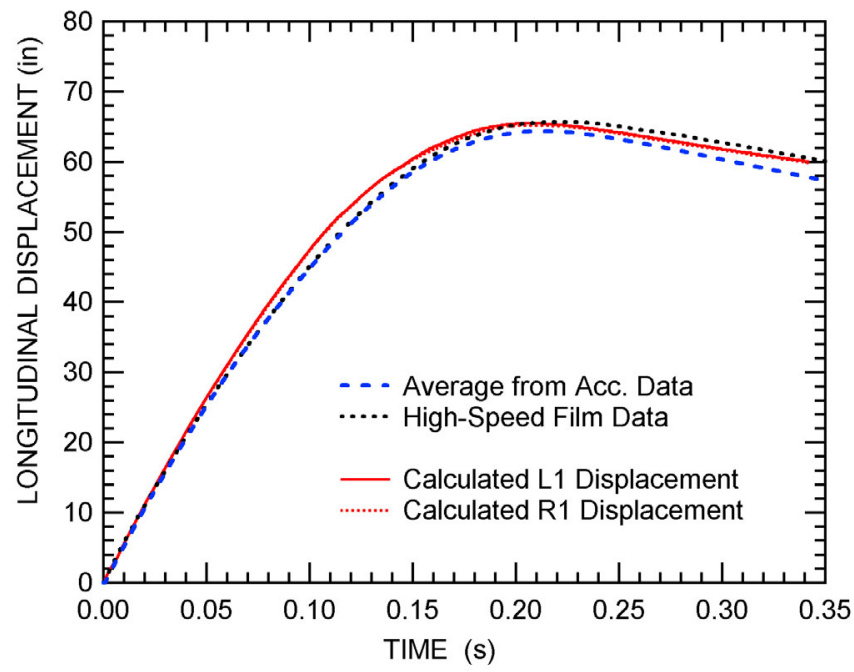

Figure 7. Comparison of the Calculated and Measured Longitudinal Crush Displacements.

The calculated collision response is compared to the test in Figure 8. The figure shows the vehicle position near the peak of the crush response. The model captures many of the significant features of the measured collision response. The vestibule region of the car is completely crushed and the damage extends into the forward region of the occupant compartment. The collision dynamics result in an upward lift of the car body that lifts the forward truck off the track. The front of the carbody was calculated to lift vertically by approximately 8 inches during the collision as compared to the measured lift of approximately 5 inches.
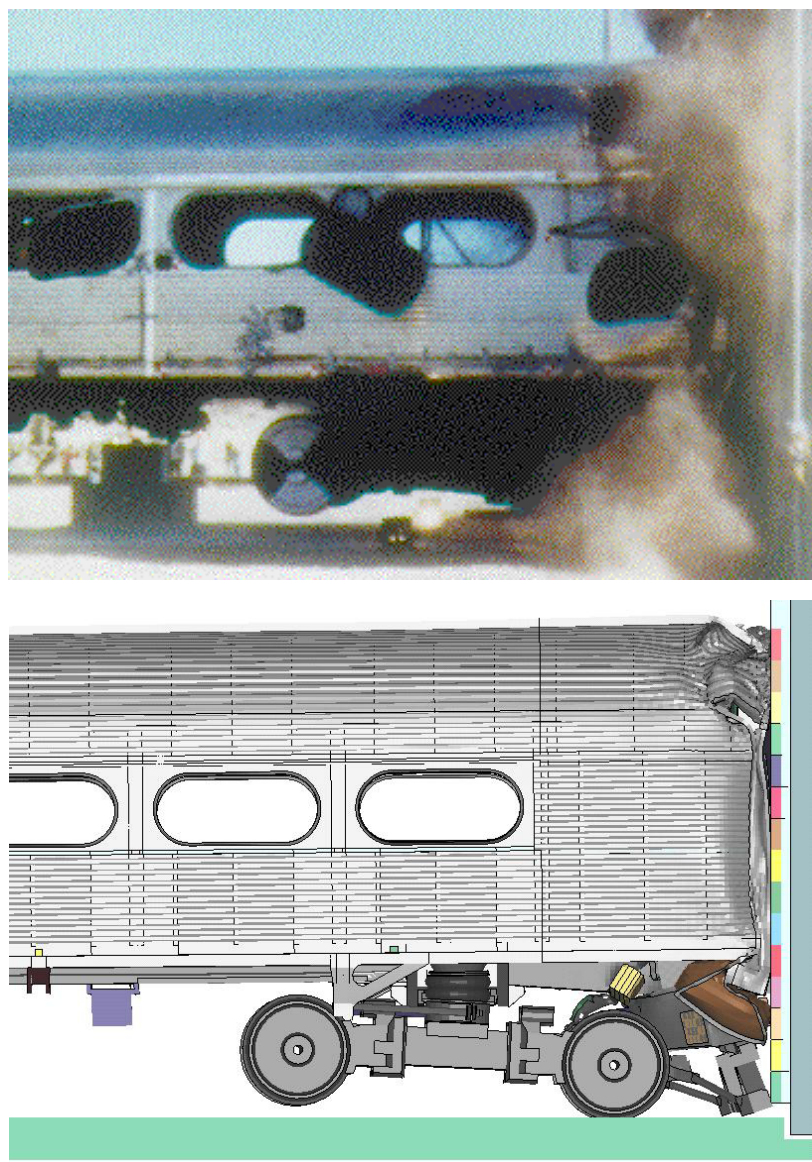

Figure 8. Measured and Calculated 35-mph Rigid Wall Crash Response.

The ability of the model to correctly capture the collapse behavior of the draft sill is important for modeling the crush response of the vehicle. Capturing the details of the collapse mode is difficult since the structure was designed to withstand a high buff load without damage rather than collapse in a controlled behavior. In this strength design approach, the most efficient design has a relatively uniform strength throughout the structure. As a result, multiple collapse modes are possible once the strength is exceeded. The specifics of the collapse modes can be changed by small differences in the local geometry or by variability in the loading conditions.

A comparison of the measured and calculated draft sill collapse modes are shown in Figure 9. In both cases, the draft gear is driven back by the collision forces and fails the connection between the buff stop to the draft sill. This initiates a collapse in the draft sill side immediately aft of the buff stop position. The forward section of the draft sill is driven back and downward as the front of the vehicle continues to crush. As the collapse proceeds, the lower plate is torn free from the 
draft sill by a failure along the weldments and the sides of the draft sill fold back on themselves. The largest difference in the measured and calculated collapse is for the left side of the draft sill where large-scale lateral buckling displacements were observed in the test and not reproduced in the model.

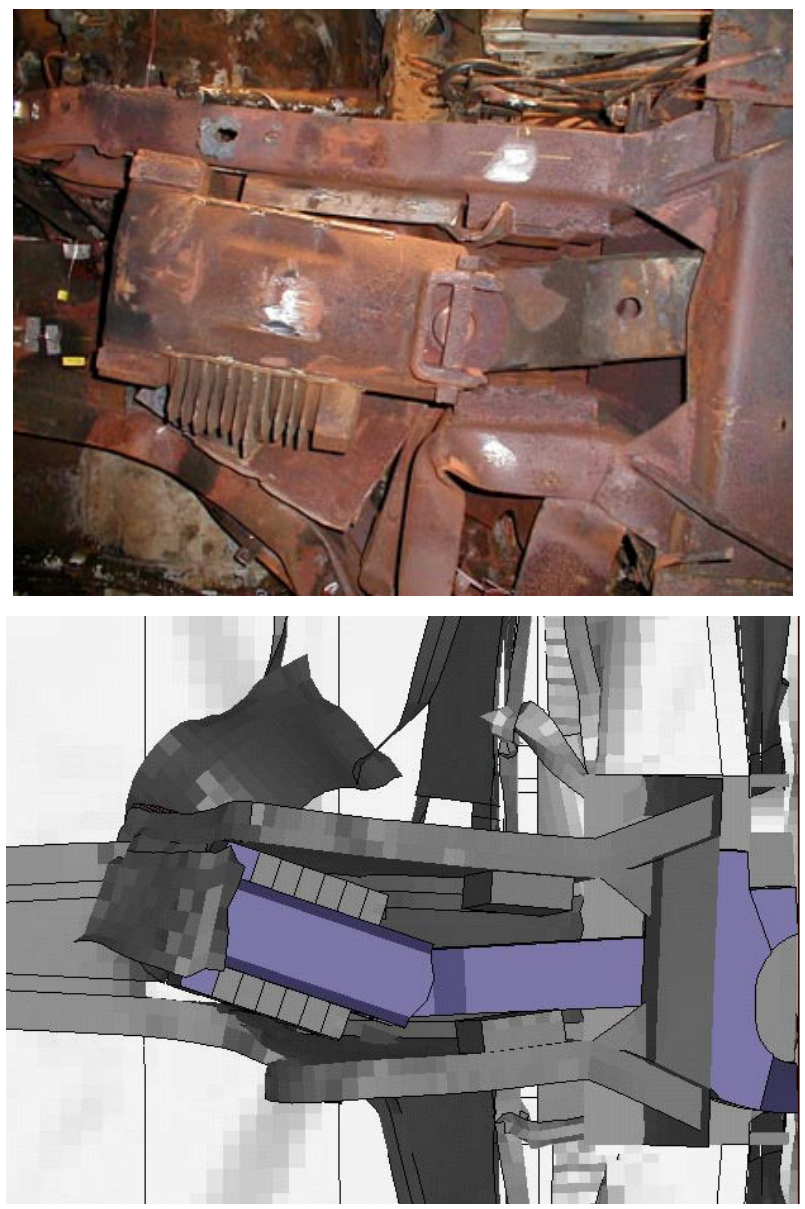

Figure 9. Comparison of the Measured and Calculated Draft Sill Collapse Modes.

Accelerometers were placed at various positions on the vehicle under frame to measure the crash accelerations as shown in Figure 10. The measured and calculated accelerations at the center sill position 2 (C2) accelerometer are shown in Figure 11. This location has potentially the closest agreement between the measured and calculated response. The magnitude of peak accelerations and overall shape of the acceleration history are similar up to a time of 0.1 second after impact. In the simulation, at approximately 0.1 second, the front section of the draft sill impacts the front axle and provides a short duration spike in the load. This short duration load spike couples into the under frame, seen as the increase in magnitude of the dynamic oscillations at that time.

In general, the agreement between the measured and calculated longitudinal accelerations is close. Two primary differences are seen in the overall comparison. First, the calculated acceleration histories in general have larger amplitude dynamic oscillations about the average acceleration pulse. The second feature that can be seen in the calculated response is the effect of the forward draft sill impact against the front truck at a time of approximately 0.1 -second. The impact of the pilot and front section of the draft sill against the truck produces an additional load path and a resulting dynamic effect that was not observed experimentally.

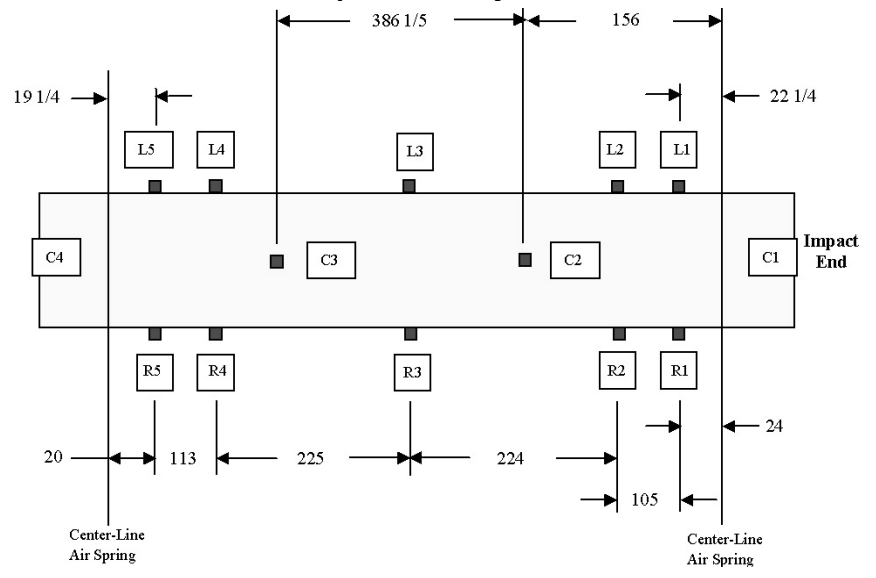

Figure 10. Test Vehicle Accelerometer Positions

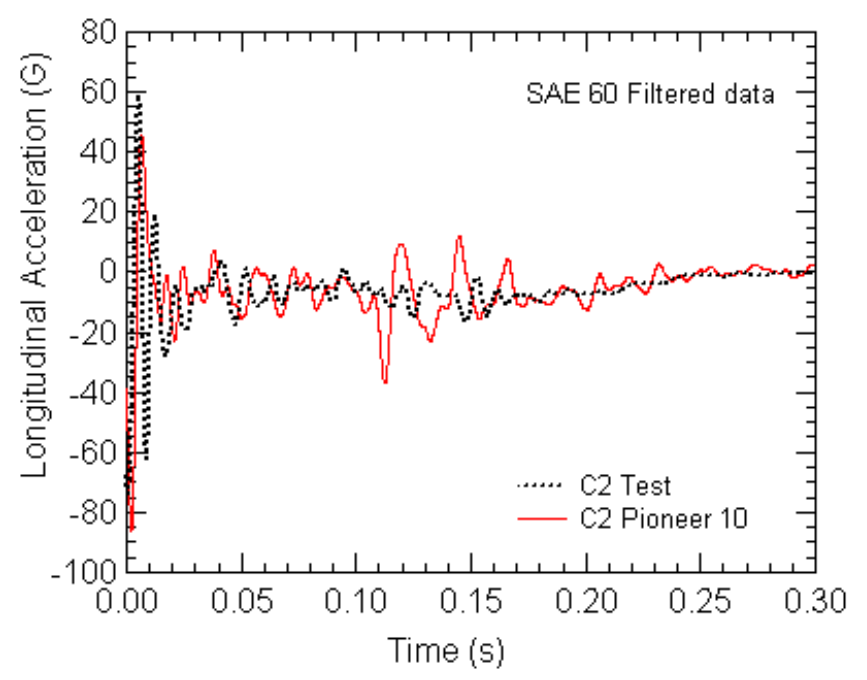

Figure 11. Calculated and Measured Center Position 2 (C2) Longitudinal Accelerations.

There are several potential sources of structural damping in the physical collision response that are not properly reproduced in the calculation. First, there are several materials in the train car that may have damping effects on high frequency behavior. These include adhesives and protective coatings on components, insulation, wood floor panels, and plastic interior wall panels. Frictional sliding between adjacent components would also dissipate higher frequency vibrations in the car body structures. Finally, the vehicle included many details such as equipment boxes and attachment brackets, tubing, and 
wiring that are not symmetric or periodic. These structural details would be dispersive of local oscillations in the structure.

In the model, many of these structural damping sources are either not included or are modeled with idealized behaviors. Layers of adhesives or coatings were not included. Floor panels were modeled using a bilinear elastic-plastic model. Adjacent components were often attached by merging coincident nodes such that frictional sliding could not occur in the model. Finally, many of the vehicle details such as tubing, wiring, and equipment attachment details were not included in the model. As a result, the simulation would not include many of the physical damping mechanisms that are active in the crash response.

There are also features of the numerical modeling that could contribute to the higher level of oscillations in the simulations. Algorithms used to control contact and penetration of adjacent components or failure algorithms for components and connections can introduce numerical noise into the simulations. These features of the model may also contribute to the higher level of dynamic oscillations seen in the simulations.

The comparisons of the measured and calculated car body longitudinal accelerations on the left side sill at positions L2 and L4 are shown in Figure 12 and Figure 13, respectively. In general the agreement between the measured and calculated accelerations is close, with the exception of the larger amplitude dynamic oscillations in the simulation. The effect of the forward draft sill impact against the front truck in the simulation can also be seen.

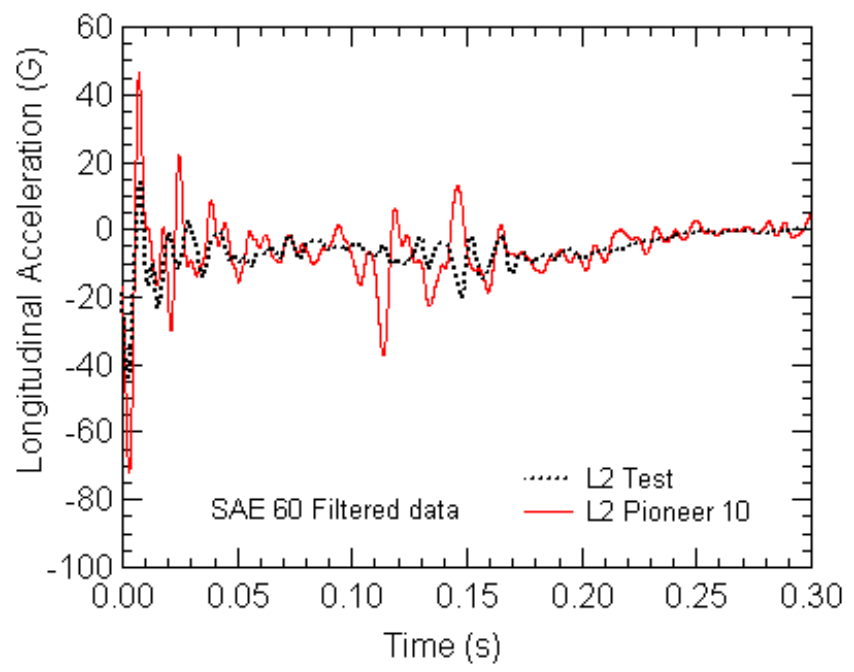

Figure 12. Calculated and Measured Left Position 2 (L2) Longitudinal Accelerations.

A comparison was made for the measured and calculated longitudinal strains at various locations on the test vehicle. The comparison of strains in a vehicle collision response can be difficult because of the influence that the local deformations have on the measured strain. The magnitude of the measured strain can vary significantly with proximity to a buckle formation. However, the overall timing and character of the strain history can provide useful data.

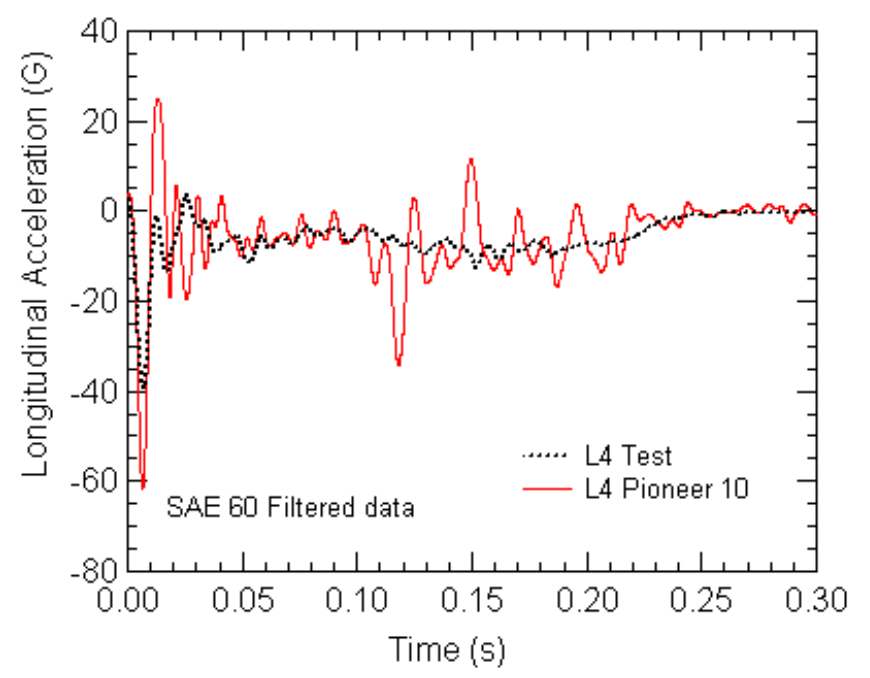

Figure 13. Calculated and Measured Left Position 4 (L4) Longitudinal Accelerations.

Close correlation was obtained between the measured and calculated draft sill strains at some draft sill locations. The measured and calculated strain histories at the right forward draft sill location are shown in Figure 14. The close correlation probably results from the similar behavior of the draft sill collapse on the right side in both the calculation and simulation. In both, the forward draft sill section is pushed back and down without large-scale structural deformations.

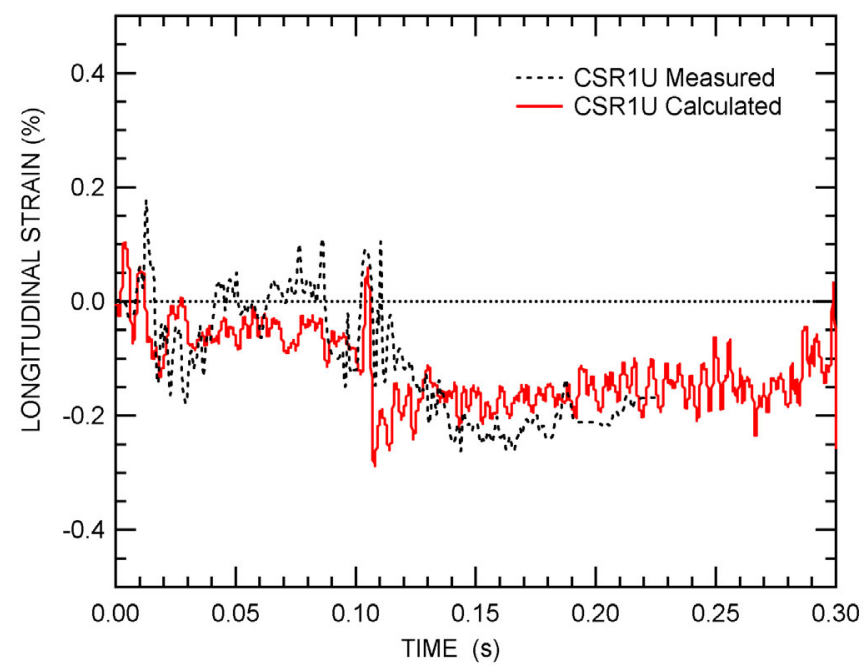

Figure 14. Calculated and Measured Right Upper Draft Sill Strains at Position 1.

The measured and calculated longitudinal force-crush histories are compared in Figure 15. The comparison shows general agreement between the measured and calculated car body crush. One of the largest discrepancies is a large spike in 
the calculated response at approximately 50 inches of crush. This spike was produced when the pilot on the underside of the draft sill impacted the front axle on the forward truck providing a direct load path to the wall.

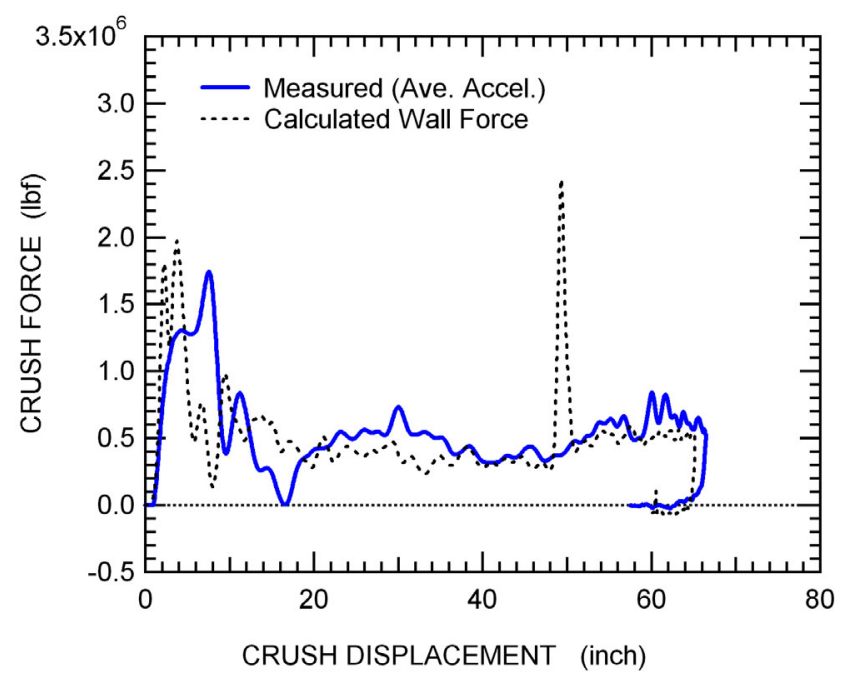

Figure 15. Measured and Calculated Crush Force Displacement Behaviors.

\section{CONCLUSIONS}

A detailed model was developed to predict the detailed car crush response as well as the three-dimensional gross motions of the car. The model developed for the Pioneer car consisted of approximately 500,000 elements. The model included several aspects not included in the previous models such as detailed modeling of the draft gear and the vehicle secondary suspension. These features of the vehicle structure were important to properly capture both the structural collapse modes and the car body gross motions.

The Pioneer car model was used to calculate the collision behavior in the single car impact test. The model captures many of the significant features of the measured collision response. The crush distance is accurately calculated and the modes of structural collapse are reproduced in the calculation. The vestibule region of the car is completely crushed and the damage extends into the forward region of the occupant compartment. The collision dynamics result in an upward lift of the car body that lifts the forward truck off the track.

\section{ACKNOWLEDGEMENTS}

The work described in this paper was performed under contract to the Volpe Center, as part of the Equipment Safety Research Program sponsored by the Federal Railroad Administration (FRA). The contract was initiated and monitored by David Tyrell and Kristine Severson, Senior Engineers, Structures and Dynamics Division, Volpe Center. Tom Tsai, Program Manager, Equipment and Operating Practices Research Division, FRA, manages the passenger rail equipment crashworthiness research.

\section{REFERENCES}

1. Tyrell, D., Severson, K., Perlman, A.B., March, 2000, "Single Passenger Rail Car Impact Test Volume I: Overview and Selected Results," US Department of Transportation, DOT/FRA/ORD-00/02.1.

2. VanIngen-Dunn, C., March 2000, "Single Passenger Rail Car Impact Test Volume II: Summary of Occupant Protection Program," US Department of Transportation, DOT/FRA/ORD-00/02.2.

3. Brickle, B., May 2000, "Single Passenger Rail Car Impact Test Volume III: Test Procedures, Instrumentation, and Data," US Department of Transportation, DOT/FRA/ORD-00/02.2.

4. Tyrell, D., Severson, K., Perlman, A.B., Brickle, B., VanIngen-Dunn, C., "Rail Passenger Equipment Crashworthiness Testing Requirements and Implementation," presented at the 2000 International Mechanical Engineering Congress and Exposition, November 6, 2000, Orlando, Florida.

5. Kirkpatrick, S.W. MacNeill, R.A. and Schroeder, M., "Development of a Computer Model for Prediction of Car Crush and Gross Motion of a Railroad Passenger Car Under Collision Conditions," Draft Final Report, Volpe National Transportation Systems Center, 2001.

6. MacNeill, R.A. and Kirkpatrick, S.W., "Vehicle Postmortem and Data Analysis of a Passenger Rail Car Collision Test," Proceedings of: JRC2002, The 2002 ASME/IEEE Joint Rail Conference, Washington D.C., April 23-25, 2002.

7. Simons, J.W. and Kirkpatrick, S.W., "High-Speed Passenger Train Crashworthiness and Occupant Survivability," International Journal of Crashworthiness, IJCrash Vol. 4, No. 2, pp. 121-132, 1999.

8. "LS-DYNA User's Manual: Nonlinear Dynamic Analysis of Structures in Three Dimensions," Livermore Software Technology Corporation, Version 940, June 1, 1997. 